exudation and consequent pressure, this will be relieved. It is this action that explains the relief brought about by radiation of the appropriate nerve roots in cases of sciatica or other neuritis, and also that latest treatment of nephritis where the use of deep rays has had the same result as a decapsulation, by destroying the leucocytes and so relieving the pressure.

From the patient's point of view the actual treatment is entirely free from pain or other sensation. Naturally a weariness is induced from remaining for the required time in one position. Prolonged exposures to the thorax and upper abdomen cause a varying degree of vomiting, which rarely lasts more than twenty-four hours. Careful preparation, the administration of chloretone, and the use of "drop enemas" of saline solution usually relieve the symptom in a short time. For some days after the treatment patients may experience a certain amount of malaise, but this does not occur in the majority. If the exposures have been correct the skin shows nothing but an erythema and a subsequent bronzing. If there be irritation it can be relieved by ointments.

There is still much to learn, and if we are to learn there must be more co-operation between the different branches of our profession in the treatment of cancer. Such treatment-as also that of fibromyomata-is to-day no longer purely surgical, and the patient has a right to expect that every means that can alleviate or cure him should be called into requisition where available. A surgeon remarked that a radiologist ought also to be a surgeon. In my opinion the boot is on the other leg. Every radiologist has learnt some surgery, and has seen more or less of it in the ordinary course of his work. The surgeon of to-day-at any rate those in middle life-never learnt anything of radiology beyond a certain acquaintance with the reading of $x$-ray pictures. It is they who have to study the developments and potentialities of the science of modern radiotherapy, and to know when to advise it; and they have far more to learn in this, the youngest of the healing arts, than the radiologist has to learn of the art of surgery; its results in the treatment of malignant disease have not kept pace with the advances in other departments of their art.

\section{A HALOMETER.}

BY

ROBERT HENRY ELLIOT, M.D., F.R.C.S., Lieutenant-Colonel I.M.S.(RET.).

WE have been steadily learning that it is important to be able to ascertain with some degree of accuracy the angular measurement of the halos or coloured rings of which patients from time to time complain; for it is becoming clear that by this means we have an important aid to differential diagnosis.

Inasmuch as the so-called physiological halos (those produced by the histological peculiarities of the lens structure) may be taken as practically constant at $7^{\circ}$, whilst the true glaucoma halos vary from time to time even in the same patient, and have a range (in my experience) of from $7^{\circ}$ or less up to nearly $12^{\circ}$, it is obviously important that we should have some ready means of measuring all such halos quickly and accurately. The method with a candle, a cardboard strip, and a pencil is clumsy and unsatisfactory. The device of having two points of light at a fixed distance showing through a metal disc-(1) a large one for the creation of the halo, and (2) a small one whereby any particular ring can be located-has this great disadvantage, that the smaller light aperture itself gives rise to halos and so may greatly confuse the patient; it is also a clumsy expedient at the best to be constantly altering the observation distance. The instrument now about to be described does away with these difficulties and makes the measurement of a halo a quick, simple, and easy proctdure which barely takes a minute to carry out, and which needs no mathematical knowledge whatever. The halometer, as I have called it, has been made for me by Mr. Clement Clarke, of 16, Wigmore Street, and I am much indebted to him for the trouble and ingenuity he has put into it.
Description of Apparatus.

A circular wooden base (B), for fitting to the wall, carries a base box (BB) to which are affixed twin aluminium bars ( $A$ and AA). This box revolves round the base through $180^{\circ}$; the object of this movement is to enable the observer to measure diagonally placed segments of halos. The box also encloses a 4-volt lamp for the principal light, which is seen through a 7-millimetre aperture (PL); this has been found a convenient diameter for the easy observation of halos.

To the left-hand bar (A) is attached a sliding box carrier (C), easily movable by the hand. Enclosed in this box is another 4-volt lamp, the light from which is trapped by a tapered glass rod (G), which is serrated, flattened, and bevelled to give the effect of a luminous grid, from which no halos are given off. This rod extends over the width of the bar. The observer slides this up or down until it corresponds with the circle of colour which it is desired to measure; since it gives off no halos, and is quite distinct in the dark, it is very easy for the patient to say exactly when the right position is reached.

The right-hand bar (AA) is engraved with an inch scale in numerals, giving the radius of any numerals, giving the radius of any
circle represented by a coloured ring. Knowing the distance $(d)$ of the eye from the source of light, and the radius $(r)$ of the halo circle as measured by the instru. ment, the angle $(a)$ subtended by this radius with the eye can be at once calculated from the relation$\operatorname{ship} \frac{r}{d}=\tan a$. On doubling the result so obtained we have the angular measurement of the diameter of the halo. In order to save calculation this has been reckoned out for measurements taken at 100 inches, and shown alongside of the wide scale in Roman figures; no mathematical mental process is necessary so long as this instrument is used at 100 inches. Three spring indicators

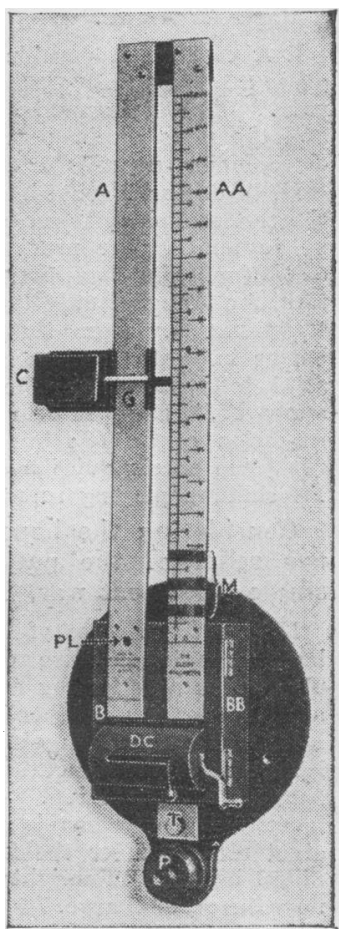

(M), coloured red, green, and blue respectively, are attached in order to register the position of the corresponding halos in the dark.

The distance of the patient from the instrument (100 inches) is controlled by a length of cord, which is wound on an enclosed drum fitted to the base box (DC); the cord can be very quickly rewound when done with.

All electrical contacts are arranged without wire or flex. The lamps are standard size. Spare lamps and trap rods are provided in the base box. All parts fitted are easily accessible.

A plug intake for current is fitted for use with a 4-volt accumulator or a dry battery, or through a resistance from the main supply.

An alternative pattern with a dry battery fitted in the base box can also be supplied if desired.

I have had this instrument at work in my consulting room for several months and have found it quite satisfactory; it has furnished valuable clinical indications.

\section{THE MECHANICS OF THE ANTERIOR ABDOMINAL WALL.}

BY

DAVID WATERSTON, M.D., F.R.C.S.ED., BCTE PROFESSOR OF ANATOMY, ST. ANDREW'S UNIVERSITY.

THE aponeuroses of the lateral muscles of the abdominal wall constitute an element mechanically of great importance in the structure of the anterior wall of the abdomen. The union and the interlacing of the fibres of these aponeuroses are well known, but $I$ have observed a mechanical feature which has not been described and one which is of considerable interest and possibly of practical importance.

The feature, briefly, is this, that the interlacement of the aponeuroses produces a fibrous membrane which is elastio 